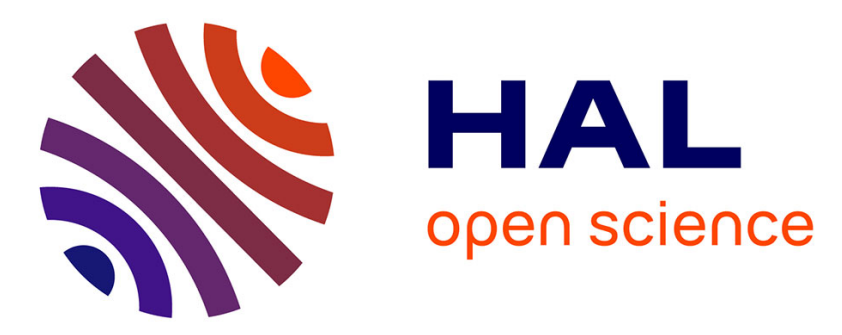

\title{
Un instrument symbolique de la domestication: le jardin zoologique aux XIXe-XXe siècles (l'exemple du parc de la Tête d'Or à Lyon)
}

Eric Baratay

\section{- To cite this version:}

Eric Baratay. Un instrument symbolique de la domestication: le jardin zoologique aux XIXe-XXe siècles (l'exemple du parc de la Tête d'Or à Lyon). Cahiers d'histoire, 1997, 42 (3-4), pp.677-706. 10.4000/ch.314 . hal-00485478v2

\section{HAL Id: hal-00485478 \\ https://univ-lyon3.hal.science/hal-00485478v2}

Submitted on 30 Jan 2011

HAL is a multi-disciplinary open access archive for the deposit and dissemination of scientific research documents, whether they are published or not. The documents may come from teaching and research institutions in France or abroad, or from public or private research centers.
L'archive ouverte pluridisciplinaire HAL, est destinée au dépôt et à la diffusion de documents scientifiques de niveau recherche, publiés ou non, émanant des établissements d'enseignement et de recherche français ou étrangers, des laboratoires publics ou privés. 


\title{
UN INSTRUMENT SYMBOLIQUE DE LA DOMESTICATION: LE JARDIN ZOOLOGIQUE AUX XIXe_XX ${ }^{\mathbf{e}}$ SIECLES (L'EXEMPLE DU PARC DE LA TÊTE D'OR À LYON)
}

\author{
Éric BARA TA y
}

Résumé : L'histoire des jardins zoologiques est à peine ébauchée alors qu'ils représentent un lieu symbolique de la rencontre entre l'homme et l'animal sauvage et un instrument de domestication. Créé en 1859 sous la forme d'un jardin d'ornement, le parc de la Tête-d'Or prit lentement le visage d'une ménagerie exotique, grâce aux dons des coloniaux et des voyageurs. Il servit bien moins à la science, en dépit des projets, qu'à la distraction du public, mais sa mise en scène affirme une volonté de maîtrise de la nature, révélatrice des ambitions de l'Occident contemporain.

Abstract: The study of zoological gardens has hardly started and yet they already reflect both a symbolic meeting point for man and the wild animal, and an instrument of domestication. Created in 1859 as a pleasure garden, the Parc de la Tête d'Or gradually took on a new orientation, that of an exotic menagerie built up through gifts from colonial and travellers. Despite the added potential, the Park was more of a public attraction than a major scientific focus. The zoological layout nevertheless confirms a desire to interact with nature, a desire wich clearly reveals the ambition of the contemporary western world.

L'auteur/the author: Maître de conférences en histoire contemporaine, Université Jean Moulin-Lyon 3.

Adresse/Address : Université Jean-Moulin-Lyon 3, 74, rue Pasteur, 69365 Lyon Cedex 07, France.

Tél. : 0472724594 et Fax : 0472722161 


\section{LESJARDINS ZOOLOGIQUES ONT UNE HISTOIRE 1}

Le terme de jardin zoologique est apparu dans la première moitié du $\mathrm{XIX}^{\mathrm{e}}$ siècle, autour des années 1830 en Grande-Bretagne et en France 2, mais il était alors peu employé et se voyait souvent confondu avec le concept plus ancien de ménagerie, que Larousse explicite comme un «lieu où l'on rassemble des animaux rares et précieux », alors qu'il ne définit pas le jardin zoologique 3. Il n'est pas possible de faire ici une histoire des ménageries. Disons seulement qu'elles existaient dès l'époque antique, auprès de certains monarques - en Chine, en Égypte, à Rome ... - pour des raisons de prestige ou de distraction (combats d'animaux), qu'elles se perpétuèrent, souvent pour les mêmes raisons, dans les cours de l'Occident médiéval puis des temps modernes, par exemple dans les principautés italiennes de la Renaissance ou à Versailles à partir de 1662-1664 lenfermant les divers éléments de la ferme, y compris les bêtes, prit le sens moderne de lieu contenant des animaux de toutes sortes d,especes, Il' p us souvent exotIques ou rares ${ }^{5}$.

De ce point de vue, rien ne distingue le jardin zoologique de l'époque contemporaine de la ménagerie des Temps Modernes, si ce n'est une présence accrue des animaux jugés spectaculaires, c'est-à-dire les «féroces» - lions, tigres .. -, les grands herbivores - rhinocéros, girafes - et les singes. Nos recherches 6 nous ont montré l'existence d'un autre point commun: la mise en spectacle des animaux au profit du public, qui apparut à l'époque de la théâtralisation baroque, d'abord en Italie (par exemple à la Villa Borghèse à Rome) puis au temps du premier Versailles de Louis XIV, marqué par

1. Robert Delort me pardonnera, je l'espère, d'avoir tenté, et réussi, le clonage de son titre: Robert DELORT, Les animaux ont une histoire, Paris, Éditions du Seuil, 1984.

2. Voir, pour la Grande-Bretagne: Wilfrid BLUNT, The Ark in the Park. The Zoo in the Nineteenth Century, Londres, Hamish Hamilton, 1976, p. 32.

3. Grand dictionnaire universel du XIX' siècle (1866-1876), Nîmes, Lacour, 1891, article «ménagerie ».

4. Le cas romain a été étudié très tôt: G. JENNISON, AnimaIs for Shaw and Pleasure in Ancient Rome, Manchester, Manchester University Press, 1937; Jacques A YMARD, Essai sur les chasses romaines des origines à la fin du siècle des Antonins, Paris, De, Boccard, 1951. On trouvera une approche nouvelle dans: David BOMGARDNER, «The trade in wild beasts for roman spectacles: a green perspective », dans Anthropozoologica, 16, 1992, pp. 161-166.

5. Voir l'histoire du mot et du concept dans: Bettina PAUST, Studien zur barocken menagerie im deutschsprachigen raum, Worms, Wernesche Verlagsgesellchaft, 1996, pp. $15-22$.

,.6. Nous préparons avec Élisabeth Hardouin-Fugier une Histoire des ménageries et jardins zoologiques du XVI! siècle à nos jours, à paraître à Paris, Éditions La Découverte, fin 1998. 
l'italianisme 7, et qui fut adoptée un peu partout aux siècles suivants.

Le jardin zoologique se différencie de la ménagerie, presque toujours princière, par son statut - national, municipal ou privé: sociétés d'actionnaires -, sa destination première -la récréation d'un public citadin indifférencié et non plus d'une cour 8 - et son inscription dans l'environnement. Alors que les ménageries médiévales étaient dispersées en divers endroits des châteaux et que celles des XVIIe-XVIII" siècles rassemblaient fréquemment les animaux en un lieu clos situé dans un jardin à la française tout en étant bien séparé des autres éléments, le jardin zoologique se caractérise, au contraire, comme son nom l'indique, par une intégration étroite de l'espace des animaux dans un parc organisé le plus souvent à l'anglaise.

Il peut donc se définir comme un jardin à l'anglaise présentant au public, en des proportions variables selon le lieu et le temps, des animaux sauvages ou subissant une action de domestication 9, exotiques ou indigènes, rares ou non. En ce sens, le premier en Occident fut celui du Jardin des Plantes à Paris, ouvert en 1793. Il servit longtemps de modèle au XIX ${ }^{\mathrm{e}}$ siècle, une époque marquée par de nombreuses créations, en vagues successives: d'abord au Royaume-Uni, à Londres (1828), Dublin (1831), Bristol (1835), Manchester (1836), etc., puis dans les capitales et les grandes villes du continent au milieu du siècle (Anvers 1843, Berlin 1844, Bruxelles 1850, Turin 1859, Budapest 1865, etc.), tandis que commençait un essaimage en France (Marseille 1854, Lyon 1858, Mulhouse 1868) et en Allemagne après 1870. Une autre phase de créations se situe dans les années 1950-1980, souvent en dehors des villes, sur des superficies élargies, à l'époque des loisirs, du tourisme et de l'automobile. C'est alors que se généralisèrent de nouvelles dénominations, telles que "parc zoologique )', qui suggère l'augmentation des échelles, ou «zoo », abrégé de jardin zoologique, qui évoque un autre glissement : une dissociation plus ou moins prononcée avec le jardin arboré et une priorité accordée à l'animal. Il reste que les trois termes sont encore largement synonymes, et que l'on peut employer d'une manière générique celui de jardin zoologique pour désigner, aux $\mathrm{XIXe-XX}{ }^{\mathrm{e}}$ siècles, un espace de présentation d'animaux le plus souvent sauvages, exotiques ou rares.

L'histoire universitaire de l'animal étant à ses débuts, on comprendra que celle des jardins zoologiques soit quasiment inexistante en France, et juste

7. Beata DI GADDO, Villa Borghese. Il giardino e le architetture, Rome, Officina Edizioni, 1985; Alfred MARIE et Jeanne MARIE, Versailles au temps de Louis XIV, Paris, Imprimerie nationale, 1976, pp. 192-193. Voir: Jean-François DUBOST , La France italienne, XV/XVI! siècle, Paris, Aubier, 1997.

8. À Versailles, le public pouvait visiter à l'occasion la ménagerie, mais celle-ci était bien destinée au roi et à sa cour.

9. Sur cette notion, préférable à celle, plus statique et proche de la Genèse, d'animaux domestiques, voir: Jean-Pierre DIGARD, L'homme et les animaux domestiques, Paris, Librairie Arthème Fayard, 1990. 
ébauchée à l'étranger, alors qu'il s'agit d'un lieu hautement symbolique dans la confrontation de l'homme et de l'animal. Ici comme ailleurs, l'initiative est venue des hommes de terrain. Ainsi, Gustave Loisel, zoologue, directeur de laboratoire à l'École des hautes études de Paris, publia le premier une Histoire des ménageries de l'Antiquité à nos jours 10. Précise, sérieuse, bien documentée et abondante, elle reste la référence, mais elle est très descriptive, peu analytique et se limite trop souvent à une nomenclature d'établissements. Par la suite sont parus des ouvrages consacrés à l'histoire de tel ou tel zoo, écrits par leurs directeurs ou par des érudits locaux, généralement à l'occasion d'un anniversaire. Riches en informations, souvent de première main, ils ont quelquefois l'inconvénient d'être construits sur un mode trop chronologique, voire anecdotique 11. Plus récemment, des historiens de l'art, des zoologues se sont intéressés à des aspects précis: inscription dans l'urbanisme, évolution de l'architecture, effets de l'arrivée de tel ou tel animal sur l'imaginaire des populations, etc. 12. Mais il n'existe pas de travaux essayant de traiter l'objet dans sa globalité.

Car différents niveaux de lecture sont possibles et plusieurs histoires s'entrelacent autour de ce sujet microcosme:

-l'histoire des jardins: au $\mathrm{XIX}^{\mathrm{e}}$ siècle, les zoos étaient avant tout des jardins et leur constitution avait été liée à ce bouleversement de la seconde moitié du XVIIIe siècle qui avait vu le modèle anglais supplanter peu à peu le type français. Le jardin à l'anglaise visant à la recréation d'une nature vraie, idéale, avec des éléments «sauvages» savamment juxtaposés, les animaux furent souvent utilisés pour l'agrémenter, pour former un "parc animé ». Il faudrait étudier cette manière d'enrichir le jardin, de présenter les bêtes, et souligner l'inversion qui s'effectua dans les zoos nouveaux ou rénovés du $\mathrm{XX}^{\mathrm{e}}$ siècle, la végétation servant désormais à mettre les animaux en perspective.

10. Gustave LOISEL, Histoire des ménageries de l'Antiquité à nos jours, Paris, Laurens, 1912, 3 volumes.

11. Quelques exemples: Il giardino zoologico di Roma nel XXVanniversario, 1910·1935, Rome, Fratelli Palombi, 1935; Centenaire du parc zoologique et botanique.- Bulletin de la Société industrielle de Mulhouse, 1968, nO 1; Annick BRAUMAN et Marie DEMANET, Le parc Léopold 1850-1950. Le zoo, la cité scientifique et la ville, Bruxelles, Archives d'architecture moderne, 1985; Yves LAISSUS, Les animaux du Muséum, 1793.1993, Paris, Imprimerie nationale, 1993. Ernest Hamy, anthropologue au Muséum, avait ouvert la voie avec ses travaux, plus restreints que ceux de Loisel: Ernest HAMY, Les anciennes ménageries royales et la ménagerie nationale fondée le 14 Brumaire an II, Paris, Imprimerie nationale, 1893.

12. Respectivement: Anna MAROTT A, Il Real Giardino Zoologico un museo naturalistico nella Torino postunitaria.- Storia dell'urbanistica piemonte, numéro spécial, juillet-décembre 1989; Bettina PAUST, Studien zur barocken menagerie ..., ouv. cité; Wilfrid BLUNT, The Ark in the Park ..., ouv. cité. 
- L'histoire de l'urbanisme: la plupart des jardins zoologiques du $\mathrm{XIX}^{\mathrm{e}}$ siècle furent installés à l'immédiate périphérie des villes. Ils représentèrent, dans la pensée hygiéniste du temps, un poumon vert destiné à régénérer un espace citadin conçu comme un organisme vivant. Ils participèrent aux grandes transformations urbaines de l'époque par lesquelles les villes s'aérèrent et s'ouvrirent à la nature - jardins mais aussi squares, quais, boulevards complantés -, mais une nature recréée, domestiquée, idéalisée, pour qu'on acceptât de l'introduire. La création des jardins s'accompagna souvent d'opérations immobilières adjacentes, facteurs d'une redistribution géographique de la population. Des lotissements résidentiels attirèrent une aristocratie et une bourgeoisie locales, de plus en plus incommodées par les miasmes urbains et la proximité sociale.

- L'histoire des collections, de la science et de la vulgarisation: la fondation des jardins zoologiques s'inscrit dans un mouvement pluriséculaire marqué par la création de collections d'histoire naturelle à partir des $\mathrm{XV}^{\mathrm{e}}$ $\mathrm{XVf}$ siècles, par le développement des explorations, par la constitution de la zoologie systématique au XVIIIe siècle et les expériences d'hybridation et d'acclimatation au XIX ${ }^{\mathrm{e}}$ siècle. Les fondateurs de jardins zoologiques eurent souvent pour premier objectif de participer à cette aventure et de nombreuses ouvertures furent parallèles à celles de muséums d'histoire naturelle. À partir de la fin du XIX $\mathrm{X}^{\mathrm{e}}$ siècle, l'accent fut plutôt mis sur la nécessaire vulgarisation des connaissances, accompagnant les progrès de la scolarisation, puis de nos jours, à l'époque de l'écologie, sur la participation à la sauvegarde des espèces. Reste à voir si la collaboration des zoos fut aussi réelle et féconde qu'il n'est dit fréquemment.

- Une histoire sociale: l'étude des jardins zoologiques doit aussi se faire sous l'angle de leurs «consommateurs ». Une analyse des fondateurs, des utilisateurs - employés, scientifiques, artistes, public -, et de leurs motivations, débouche sur une histoire de la philanthropie, de l'instruction, de la curiosité, des loisirs et du tourisme. Une histoire qui doit être différenciée, chaque groupe social ayant sa lecture de l'espace zoologique et ses points d'attraction privilégiés.

- L'histoire de l'architecture: les historiens de l'art découvrent à peine ces bâtiments, longtemps jugés mineurs. Pourtant, de l'architecture industrielle du zoo de Bruxelles en 1850, au rocher des singes cubiste de Mulhouse en passant par la maison des herbivores «art déco» de Bâle, les éléments origiriaux sont nombreux. Ils montrent un hiatus entre la prétention scientifique des jardins et une architecture éclectique jamais adaptée à l'animal, mais qui illustre la volonté d'autonomie des architectes, l'aspect primordial de la mise en spectacle, la participation des zoos à la domestication de la nature en l'obligeant à se fondre dans un produit de civilisation.

L'histoire de la relation à l'animal: nous arrivons ainsi à une question rarement abordée dans les histoires des jardins, ou alors d'une 
manière anecdotique. Le zoo est un lieu clos de confrontation de l'homme et de l'animal, un endroit idéal pour lire les préférences du premier, les manières de présenter, de considérer et de traiter le second, leurs stratifications sociales et leurs évolutions. Plus largement, en rassemblant la faune et la flore des quatre continents, le zoo est un moyen réel, mais surtout symbolique, de s'approprier la nature, voire de la domestiquer, c'est-à-dire de l'introduire dans la domus, dans l'espace humain. En ce sens, la constitution et la diffusion des jardins ont incarné et facilité l'entreprise occidentale de maîtrise du monde, commencée avec les grandes découvertes.

Le but de cet article est d'expérimenter, sur un exemple concret, la fécondité du sujet et la variété des approches, mais aussi de montrer la participation du zoo à l'appropriation du monde sauvage, à la volonté plus ou moins consciente de le domestiquer. Le parc de la Tête d'Or, créé en 1858 à Lyon, appartient à la seconde génération des jardins zoologiques du $\mathrm{XIX}^{\mathrm{e}}$ siècle. Il présente le triple avantage de permettre une étude sur la longue durée, de laisser voir, par son statut municipal, la diversité des acteurs, et de posséder des archives abondantes, plus riches qu'à Marseille ou Mulhouse, comparables à celles du Jardin des Plantes de Paris.

\section{LA CRÉATION DU PARC DE LA TÊTE D'OR}

Celle-ci est maintenant bien connue, c'est pourquoi nous irons vite 13. En 1856, le préfet Vaïsse, qui faisait aussi office de maire de Lyon, fit accepter par le conseil municipal la création (envisagée dès la première moitié du siècle) d'un parc public et le choix de la ferme de la Tête d'Or, située au nord-est de la ville, sur la rive gauche du Rhône annexée en 1852, déjà évoquée par l'architecte Crépet dans un projet d'embellissement de la Guillotière 14. Nommé en 1853, en même temps qu'Haussmann à Paris, et lui aussi grand promoteur d'une transformation urbaine, Vaïsse voulait sans doute imiter le bois de Boulogne alors en cours d'aménagement (1852-1860). Mais des motifs plus profonds que le mimétisme et la concurrence avec la capitale, auxquels les Lyonnais n'étaient cependant pas insensibles, entrèrent en jeu, là comme ailleurs, à Paris, Londres, Berlin 15, Bruxelles, etc.

Le premier était ce besoin de promenade, jugé indispensable par les édiles pour la récréation dominicale des classes laborieuses et pour les moments

13. Voir: Tête d'Or. Un parc d'exception créépar Denis Bülher, Lyon, CAUE, 1992; Louis-Michel NOURRY, Lyon, le parc de la Tête d'Or, Marseille, AGEP, 1992.

14. Arch. mun. Lyon (Archives Municipales de Lyon), 925 WP 264, Procès verbal du conseil municipal du 14 mars 1856; CREPET, Notice historique et topographique sur la ville de la Guillotière, Lyon, Mailé, 1845, p. 41.

15. Où s'était imposée dès la fin du XVIIIe siècle l'idée du Volkspark, parc populaire, en opposition au Schlosspark, parc du château: Tête d'Or ..., ouv. cité, p. 3. 
de loisirs des plus aisées, l'enrichissement de la bourgeoisie permettant de multiplier les équipages, de satisfaire la volonté de paraître, de participer à des mondanités de plein air. Toutes choses qui étaient jusqu'alors limitées dans cette cité étroite, comprimée par la croissance démographique. À cela s'ajoutait le désir de quitter quelquefois la ville, de fuir la circulation, le bruit, les poussières, le voisinage des modestes. La création du jardin fut ainsi la résultante de tendances profondes parmi l'aristocratie et la bourgeoisie aisée: un souci croissant d'hygiène, une volonté naissante de séparer les vies privée et professionnelle, une réticence à la proximité sociale, auxquels s'ajoutaient sans doute les effets d'un «moi» romantique répandu dans la première moitié du siècle. Le jardin, définit par Vaïsse comme devant être retiré, calme, pur, vaste et dégagé, était aux antipodes de la ville de l'époque, et on peut se demander s'il n'était pas une projection de la cité idéale. C'est pourquoi, il s'inscrivait (troisième raison) dans ce programme de rénovation urbaine mené par Vaïsse, mais qui avait commencé dès la Monarchie de Juillet: percement de larges rues, création de places et de quais arborés, transformation des anciens remparts en boulevards. Il s'agissait de faciliter la circulation pour activer l'économie, d'ouvrir des perspectives pour divertir, d'aérer pour mieux vivre. Le jardin était l'un des éléments de cette modernité et devait contribuer, comme Vaïsse l'écrivit lui-même, à donner à la ville des allures de grande capitale. Pour tout cela, le site choisi était proche des Terreaux, le quartier privilégié de la bourgeoisie d'affaires, et relié à la ville par les nouveaux quais, tout en étant séparé d'elle par le Rhône, ce qui devait lui garantir l'isolement, la pureté, la fraîcheur et la perspective recherchés 16

Les travaux furent confiés à Denis Bühler, alors installé à Paris et spécialisé dans la création de jardins pour l'aristocratie et la grande bourgeoisie 17. On lui demanda de dessiner un parc «dans le genre paysager avec de grands effets de prairies et de groupes d'arbres, en ménageant les plus jolis points de vue des environs ». Il devait aussi comprendre les installations d'un jardin botanique. Bühler divisa l'espace en deux zones reliées par des "plantations pittoresques " : le parc paysager proprement dit, placé à l'ouest, centré sur une pièce d'eau et une colline permettant toutes les visions, sillonné d'allées, bordé à l'est par une «vallée» ombragée et des prairies; le jardin botanique, cantonné à l'est, là où les horizons étaient médiocres mais les sols de bonne qualité. Construction artificielle, miniaturisée d'une nature idéale à

16. Sur tout cet aspect: Rapport de M. le Sénateur chargé de l'administration du Rhône au conseil municipal de Lyon sur le projet de création d'une promenade publique à la ferme de la Tête.d'Or, Lyon, 1856, pp. 1-4. Pour une réflexion sur la place des jardins dans la ville: Louis-Michel NOURRY, Les jardins publics en province: espace et politique au XIX' siècle, Rennes, Presses universitaires de Rennes, 1997.

17. Arch. mun. Lyon, 485 WP 1, Bühler à préfet, 9 août 1856 et Procès verbal du conseil municipal, 14 novembre 1856. Sur Bühler et les travaux: Tête d'Or ..., ouv. cité, pp. 7-11; Louis-Michel NOURRY, Lyon ..., ouv. cité, pp. 31-56. 
la fois «sauvage» et apprivoisée par juxtaposition de divers éléments - lac, ruisseau, bois, colline -, le parc paysager proposait bien un espace calme et frais, opposé à la ville, mais en même temps relié à elle par le jeu des perspectives 18, la non-clôture, en vigueur jusqu'en 1901, et une transition assurée par le quai d'arrivée et les boulevards de bordure aux allées rectilignes complantées. Tous ces éléments concrétisaient une dialectique entre la nature et la culture, la première s'avançant peu à peu dans la ville, tout en étant de plus en plus policée, domestiquée par la seconde 19. Réalisé sur une superficie de 104 hectares (dont 63 boisés, 17 en eaux 2 , terminé en 1859, le parc accéléra l'urbanisation du quartier voisin des Brotteaux, qui attira une partie de la bourgeoisie qui privilégiait déjà, dans la presqu'île, les places, les quais, les larges rues. Là se créa une ville nouvelle, au plan en damier et aux vastes artères rectilignes, qui répondait aux désirs d'hygiène, de séparation sociale, de disjonction des lieux de vie et d'affaires 21.

Vaïsse avait prévu dès l'origine d'installer un jardin zoologique, mais il hésita sur sa forme. Il pencha d'abord pour un "parc d'animaux destinés à l'acclimatation et à la propagation des races utiles, encore peu répandues ». L'idée avait deux origines. L'ouverture du parc était d'abord l'occasion de transférer là le vieux jardin des plantes de la Croix-Rousse et de lui adjoindre une pépinière, une politique utilitariste, qu'on voulait étendre à l'animal 22. Mais ce projet était d'autant plus séduisant que l'acclimatation et la domestication des espèces exotiques étaient à la mode sous l'impulsion d'Isidore Geoffroy-Saint-Hilaire et de la Société impériale d'acclimatation qu'il venait de créer en $1854^{23}$. Pourtant, la tentation de fonder une ménagerie

18. La colline permettait la vue sur Lyon, un aspect important au moment où l'hausmanisation des grandes villes facilitait leur appropriation visuelle par leur contemplation en différents points de vue: Alain CORBIN [dir.], L'avènement des loisirs, Paris, Aubier, 1995, pp. 134-142.

19. Arch. mun. Lyon, 485 WP 1, Programme donné à Büh1er, 4 a011t1856 ; Mémoire descriptif sur le projet d'un parc paysager, octobre 1856; Ingénieur en chef à Vaisse, 29 octobre 1856.

20. M. GODIN, Documents statistiques sur les diverses branches de la voirie municipale, Lyon, Gallet, 1878, pp. 29-32.

21. Jean-Luc PINOL, Mobilités et immobilisme d'une grande ville,' Lyon de la fin $d u$ $X I X^{\prime}$ siècle à la Seconde guerre mondiale, Lyon, Centre Pierre Léon d'histoire économique.et sociale, 1989, pp. 177-194; Catherine PELLISSIER, La vie privée des notables lyonnais (XIX' siècle), Lyon, Éditions lyonnaises d'art et d'histoire, 1996, pp. 13-27.

22. Arch. mun. Lyon, 485 WP 1, Ingénieur en chef à Vaïsse, 19 février 1856; Rapport de M. le Sénateur ..., ouv. cité, p. 6.

23. Voir: Isidore GEOFFROY SAINT-HILAIRE, Acclimatation et domestication des

animaux utiles, (1861), Paris, La Maison Rustique, 1986; le Bulletin de la Société impériale d'acclimatation, à partir de 1854; Claude BLANCKAERT, «Les animaux "utiles" chez Isidore Geoffroy Saint-Hilaire: la mission sociale de la zootechnie », 
exotique, à l'instar de nombreuses villes étrangères, émergea dès 1856. Bühler alla en visiter certaines, mais il revint avec la conviction que cela coûterait cher, et ne retint que l'idée d'enclos à cerfs et à daims dans son projet définitif d'octobre 1856. Soucieux d'aménager le parc aux moindres frais, le préfet renonça à la ménagerie en 1858, et opta pour un compromis: on acheta des cerfs, des daims, des volatiles exotiques - canards, cygnes, poules, faisans, etc. - pour l'ornement, des vaches, des moutons et des chèvres exotiques, ou provenant d'autres pays européens, pour les faire connaître et montrer le parti qu'on pouvait en tirer. L'ensemble devait «animer» le paysage, renforcer l'illusion d'une vraie nature alternant campagnes et zones sauvages. L'idée n'était pas nouvelle: elle avait été énoncée par les promoteurs des jardins à l'anglaise dans la seconde moitié du XVIIIe siècle, tel le vicomte Girardin créateur du parc d'Ermenonville, et Marie-Antoinette avait fait venir fermiers et troupeaux au Hameau du Trianon pour« animer le tableau» 24.

\section{QUEL JARDIN ZOOLOGIQUE? 60 ANS D'HÉSITATIONS}

La décision du préfet ouvrit en réalité une longue période d'incertitude sur le profil exact du jardin zoologique, qui varia au gré des intervenants et des circonstances. En cela, il s'inscrivait dans une longue tradition des ménageries qui oscillaient souvent entre les deux pôles extrêmes de la simple ferme à volatiles et de l'exhibition exotique. Dans le cas lyonnais, cette situation était le fruit des hésitations d'une municipalité tiraillée entre plusieurs impératifs: ne pas dépenser trop, ni alourdir les services, tout en offrant un jardin agréable. La gestion fut donc confiée, dès 1858, à un fermier, éleveur d'animaux reproducteurs à Paris, qui devait entretenir les bêtes, les remplacer le cas échéant, et "faire des éducations» auprès du public. Il recevait une subvention, l'aide de gardiens municipaux, et la disposition des fourrages et des produits du cheptel. Mais, dès 1860 , la ville considéra que les animaux, trop peu nombreux, ne faisaient pas «d'effet décoratif», et qu'il fallait de véritables troupeaux. Au prix d'un relèvement important de la subvention, elle imposa l'entretien de près de 900 animaux - 60 bovins, 76 ovins, 21 caprins, 38 cerfs et daims, 600 animaux aquatiques et de basse-cour... -, qui devaient

dans Revue de,synthèse, 1992, nO3-4, pp. 347-382; Michael OSBORNE, Nature, the Exotic and the Science of French Colonialism, Bloomington-Indianapolis, Indiana University Press, 1994.

24. Arch. mun. Lyon, $485 \mathrm{WP} 1$, Note sur un projet de parc, 24 juin 1856; 925 WP 264, section 56, volume 1, Rapport de Bühler, septembre 1856 ; 485 WP 1, Mémoire descriptif... par Bühler, octobre 1856; 485 WP 12, Rapport de Vaïsse au conseil municipal, 1858, et Traité entre la ville de Lyon et Gérard, 26 avril 1858. R. GIRARDIN, De la composition des paysages, Paris, Delaguette, 1777; PierreAndré LABLAUDE, Lesjardins de Versailles, Paris, Scala, 1995, p. 155. 
L'administration fut confiée à la direction de la voirie, qui militait depuis longtemps pour cette solution, qui travailla par la suite à montrer que sa gestion était bonne, qu'elle coûtait moins cher que toutes les autres, et que la bonne conservation du parc était sa seule préoccupation. Mais sa politique de reconstitution du cheptel (tombé à 248 têtes fin 1875), d'amélioration de l'alimentation pour limiter les pertes et favoriser les reproductions, de rénovation des bâtiments, l'obligea à réclamer de nouvelles subventions dans un contexte contraire de baisse continue depuis 1872. L'opposition de certains conseillers municipaux à une gestion directe de la ville, sans doute par crainte d'un interventionnisme leur rappelant trop l'épisode Hénon de $1870^{29}$, leur volonté de diminuer un peu plus les fonds, obli èrent le préfet-maire à constituer une commission de réorganisation en 18760.

Celle-ci permit l'arrivée d'un nouveau groupe d'acteurs puisqu'elle compta en ses rangs le docteur Lortet, directeur du Muséum d'histoire naturelle de Lyon et professeur de zoologie à la Faculté des sciences de Lyon, ainsi que le doyen Faivre, de la même faculté, qui rédigea le pré-rapport et orienta les conclusions. Soucieuse de trouver un compromis, elle réitéra le refus de bâtir un jardin zoologique trop coûteux, et se garda de définir un objectif prioritaire. Elle réaffirma le rôle «d'embellissement et d'animation» des animaux, soutint la nécessité de maintenir des espèces «utiles» pour les faire connaître aux éleveurs, et ajouta une fonction supplémentaire à la demande des scientifiques, notamment de Lortet, qui voulait établir une synergie entre les recherches du muséum, de la faculté des sciences, des écoles vétérinaire et de médecine, et qui considérait le parc comme le terrain idéal de cette fusion. Il s'agissait de réunir et d'étudier des animaux « rares, curieux» en ouvrant des laboratoires de physiologie et d'anatomie comparée. Enfin, elle se prononça pour le retour à la gestion indirecte 31 .

L'impossibilité de trouver un gérant pour une affaire non rentable, et la campagne hostile du service de la voirie, permirent à celui-ci de garder la direction du jardin. Mais il dut vite la partager avec les scientifiques, car Lortet, qui avait déjà convaincu le conseil municipal, en 1878, de transférer le muséum

2 février 1874; 485 WP 14, Rapport de la voirie, 20 août 1873, État des Procès verbaux dressés contre Estienne; 10 juin 1874, Procès verbaux de la commission municipale, 18 novembre 1874 .

29. Porté à la mairie en septembre 1870, Hénon décida la suppression de l'octroi, la création de l'imp8t sur le revenu, l'enseignement laïc et gratuit, la séquestration des biens des congrégations et la séparation de l'Église et de l'État: Françoise BAYARD et Pierre CA YEZ [dir.], Histoire de Lyon ..., ouv. cité, p. 296.

30. Arch. mun. Lyon, 485 WP 12, Rapports voirie, 26 janvier 1875, 25 novembre 1875 ;

'. 485 WP 4, Procès verbal du conseil municipal, 28 janvier 1876; Rapports voirie, 11 mai et 23 décembre 1876.

31. Arch. mun. Lyon, 485 WP 4, Pré-rapport Faivre, 1876; Rapport de la commission, 24 octobre 1876.

Cahiers d'Histoire, nO 3-4, 1997, L'animal domestique, pp. 677-706 
près du parc, fit créer une direction scientifique en 1880: un professeur d'histoire naturelle s'occuperait désormais du jardin botanique, et lui-même du jardin zoologique. Cette division des responsabilités, technique d'un côté, scientifique de l'autre, suscita des conflits. Dès 1881, Lortet présenta un ambitieux programme d'achat d'espèces exotiques - singes, otaries, zèbres ... et de rénovation des bâtiments pour limiter la mortalité, qui nécessitait une augmentation du personnel et des subventions. La direction de la voirie souligna son côté dispendieux et milita pour un jardin modeste, limité à des animaux "robustes» d'acclimatation ou d'ornement 32.

Cette confrontation, ainsi que la politique municipale de rigueur budgétaire, obligèrent au compromis permanent et à une gestion au jour le jour avec tout de même deux constantes. La première fut le rejet unanime, de la part de l'administration et des scientifiques, des demandes de concessions émanant de personnalités extérieures, parce qu'elles les auraient dessaisis de leurs pouvoirs. Déjà, en 1874 , la direction de la voirie s'était opposée au projet d'Isidore Geoffroy Saint-Hilaire de créer un jardin annexe de celui du bois de Boulogne en soutenant, avant de dire le contraire en 1881 contre Lortet, que l'acclimatation était impossible au parc et qu'il fallait s'en tenir à l'ornement. On repoussa en 1902, et pour les mêmes raisons, une proposition similaire, qui prétendait réaliser un grand centre de vente d'animaux. Les offres de Bidel, directeur d'une ménagerie fixe, en 1876 et 1901, du cirque Bouglione en 1929 et d'un dompteur en 1930 - ils voulaient installer leurs animaux en échange de subventions et de divers privilèges d'exploitation - n'eurent pas plus de succès 33. La direction du jardin l'emporta à chaque fois, parce que les projets aboutissaient à la suppression de la gratuité - une situation rare en Europe que la municipalité envisageait avec peine. L'arrivée à la mairie d'Edouard Herriot en 1905 imposa définitivement la gestion directe, car la gratuité lui paraissait indispensable pour l'accès du public populaire.

Seconde constante: la constitution du cheptel aux moindres frais, les maigres budgets ne permettant même pas de faire face aux dépenses courantes, obligeant à mendier des rallonges en fin d'année, et surtout à ponctionner les

32. Arch. mun. Lyon, 485 WP 4, Rapport voirie, 23 décembre 1876, Procès verbal de la commission, 9 mai 1877; Lortet au maire, 10 juillet 1880; Procès verbal de la commission, 8 novembre 1880; Lortet au maire, 3 mai 1881 ; Rapport voirie, 7 mai 1881. Sur Lortet: «La vie et les travaux de Louis Charles Lortet", dans Archives du Muséum d'histoire naturelle de Lyon, tome XI, 1912, pp. 3-7; Louis DAVID, «Histoire du Muséum d'histoire naturelle de Lyon", dans Rive Gauche, nO 69, juin 1979, pp. 16-17, nO 70, septembre 1979, p. 13.

33. Arch. mun. Lyon, 485 WP 12, Rapport voirie, 17 mars 1874; De l'utilité d'un jardin zoologique d'acclimatation à Lyon, 1902; Rapport directeur des cultures, 3 décembre 1902; 485 WP 4, Bidel au préfet, 1876; 485 WP 12, Préfet au maire, 6 mars 1877 ; 485 WP 10, Bolart au maire, 29 juillet 1901; 961 WP 72, Bouglione au maire, 12 décembre 1929 ; Petit au maire, 3 novembre 1930. 
cre'd'Its $\mathrm{d}_{\mathrm{e}}$ renouve $\mathrm{Il}_{\text {ement }}{ }^{34}$. $\mathrm{J}_{\text {usqu }} \mathrm{d}$ a secon $\mathrm{d}_{\mathrm{e}}$ mOttle $\mathrm{d}_{\mathrm{u}} \mathrm{XX}^{\mathrm{e}} \operatorname{siec}^{\mathrm{l}} \mathrm{e}$, cette situation conduisit à différer les achats prévus, par exemple ceux de Lortet en 1878 , à refuser bien souvent les incessantes propositions des marchands sous prétexte d'un manque de crédits ou d'installations, et à se contenter d'acquérir des volatiles exotiques, des singes et quelquefois de gros herbivores (zèbres, chameaux) à l'hébergement plus aisé que les carnivores. Pour financer cela, la direction reprit à son compte une politique déjà pratiquée sous le Second Empire: elle vendit les progénitures des animaux les plus prolifiques - daims, pigeons, poules, cygnes ... - à des bouchers, des propriétaires de terrains de chasse, des notables désireux d'orner leurs maisons de campagne, des jardins municipaux, ces derniers devenant majoritaires dans les années 1930 alors que se diffusait leur mode dans les moyennes et les petites villes. Un autre moyen d'enrichir les troupeaux, instauré dès 1878, consista à échanger des espèces avec des importateurs-naturalistes, des éleveurs, des directeurs de jardins zoologiques ou de jardins publics, des propriétaires terriens 35 .

L'insuffisance des achats fut surtout compensée par les multiples dons au parc, qui se développèrent à partir des années 1880 à l'instar de ce qui se pratiquait déjà au Jardin des Plantes de Paris et au zoo de Marseille. Leurs origines furent diverses : des directeurs de cirques, offrant leurs bêtes vieillies, ou de jardins zoologiques (dans l'Entre-deux-guerres), des particuliers, de plus en plus nombreux à acquérir des bêtes exotiques auprès des importateurs à mesure que s'intensifiait le commerce colonial, mais qui s'en lassaient ou qui voulaient leur donner un cadre de vie plus adéquat que leurs appartements! Plus fréquents furent les dons de voyageurs - touristes aisés souvent chasseurs, industriels, commerçants, ingénieurs en tournées d'affaires, scientifiques en voyages d'études ... - partis dans les colonies et revenus avec un spécimen capturé ou acheté, faisant office de souvenir (une pratique en plein développement), à l'image des objets d'archéologie ou des tapis persans 36. Nombreux furent aussi les dons des résidants à l'étranger: militaires en postes et administrateurs coloniaux, tels le Résident général à Tunis ou les Gouverneurs généraux de Madagascar, d'Algérie, d'Indochine. La municipalité prit même l'habitude, et la présence d'Herriot facilita les démarches, de solliciter ces derniers pour obtenir un individu précis, par exemple un éléphant d'Asie en 1924. Elle dut pourtant refuser des dons par manque de place,

34. Arch. mun.,Lyon, 485 WP 4, Lortet au conseil municipal, 26 février 1886; Voirie au maire, 23 novembre 1887 ; Directeur des cultures au maire, 3 mars 1902.

35. Arch. mun. Lyon, 485 WP 4, Procès verbaux de la commission, 4 mars et 10 octobre 1878; Rapports voirie au maire, 23 décembre 1876 et 8 mars 1887; Direction des cultures au maire, 4 février 1902. On trouvera d'innombrables cas d'achats, ventes, échanges en $485 \mathrm{WP} 11$, pour 1885-1900, et $961 \mathrm{WP} 72$ pour les années 1930. Sur la diffusion des parcs privés de villégiature: Alain CORBIN [dir.], L'avènement des loisirs..., ouv. cité, pp. 90-92.

36. Voir: Alain CORBIN [dir.], L'avènement des loisirs..., ouv. cité, p. 98. 
d'installations ou de crédits de fonctionnement, une situation encore plus sensible dans les années 1930 avec la crise et la réduction des budgets.

Les dons permirent de décliner les propositions de privatisation ou celle du Muséum de Paris, en 1933, qui voulait placer des animaux en dépôt dans les jardins de ?rovince et organiser des roulements, ce qui aurait supprimé toute autonomie 3, comme ils facilitèrent la reconstitution des collections après 1918 et 1944. Ils permirent surtout une évolution progressive, inavouée, du jardin, car le choix effectué par la direction scientifique se fit de plus en plus au profit des animaux sauvages plus ou moins exotiques et aux dépens des bêtes d'acclimatation et d'élevage. Ainsi arrivèrent des ours en 1877 et 1881, des chacals en 1881, des gazelles en 1883, une panthère, des kangourous et des autruches en 1888, des crocodiles en 1890, un éléphant en 1924, des zébus en 1928 , etc. Le jardin devint une vitrine de la faune coloniale, un instrument symbolique de son appropriation - il reçut les spécimens de l'exposition coloniale qui s'était tenue au parc en 1894 -, et il se transforma peu à peu en ménagerie exotique, après avoir été surtout une ferme avant 1875, et un parc d'espèces d'ornement par la suite. Avec l'aménagement de la fauverie en 1922 et celui de la cage de l'éléphant en 1924, il prit son visage actuel, désormais semblable à celui des zoos français et européens 38 .

\section{À QUOI SERT UN JARDIN ZOOLOGIQUE?}

La volonté d'utiliser le parc pour acclimater et propager des espèces avait été affirmée dès les origines, peut-être parce que l'argument d'utilité pouvait convaincre la bourgeoisie industrieuse locale, mais surtout, nous l'avons vu, parce que l'objectif était à la mode. Il fut réaffirmé lors de la réorganisation de 1876, au nom d'un autre mot d'ordre de l'époque: la propagation du progrès. Jusqu'en 1875 , les efforts des fermiers portèrent sur la diffusion des espèces domestiques européennes, puis, sous les effets d'un optimisme ambiant dû la révolution industrielle, à la colonisation et à la croyance en un progrès indéfini, on se laissa gagner à des expériences sur les bêtes domestiques exotiques (chameau, lama ...) et surtout au rêve d'une domestication généralisée de la faune sauvage, dont le jardin zoologique serait le laboratoire privilégié. En 1887, par exemple, le parc s'intéressa aux possibilités d'élevage des jeunes chamois par des chèvres ou au dressage des zèbres du Cap'! 39

37. Arch. mun. Lyon, 963 WP 37, Muséum de Paris au maire, 2 février 1933,

38. De nombreux cas de dons dans: Arch. mun. Lyon, 485 WP 10 et 11 (1870-1900), 961 WP 72 et 73 (1920-1940). Voir les reconstitutions d'après-guerres dans: Arch.

$\sim$ mun. Lyon, 961 WP 72 (1921-1929) et 963 WP 37, Correspondance directeur du zoo-maire en 1947.

39. Arch. mun. Lyon, 485 WP 11, Rapport voirie, 28 mai 1887, Jardin d'acclimatation 
Mais, en réalité, tout cela resta très limité, pour des raisons à la fois locales et générales. Le parc, au climat froid et humide, fut jugé peu adapté. Le public ne s'y intéressa guère et la direction scientifique orienta ses choix vers des bêtes de collection. Enfin, là comme ailleurs, l'échec, l'inutilité, voire la puérilité des tentatives envers les espèces sauvages - on voulait dresser des zèbres pour ... promener le public! -, apparurent peu à peu évidents, d'autant plus que l'objectif initial de développement de l'alimentation et de la force motrice fut peu à peu atteint par l'amélioration du cheptel domestique et la mécanisation de l'agriculture, tandis que la volonté de préserver la faune sauvage prenait de l'importance au fil du $\mathrm{XX}^{\mathrm{e}}$ siècle. Au parc, on ne parla quasiment plus d'acclimatation après la Première guerre mondiale ${ }^{40}$.

L'étude scientifique des animaux, une autre fonction qu'on lui prêta souvent, n'eut guère plus d'ampleur. Elle n'avait pas été prévue à l'origine, à la différence des plantes pour lesquelles on avait construit des pépinières, une orangerie, un arboretum, des serres, etc. 41. Le transfert au parc de l'ancien jardin botanique peut expliquer cette différence initiale, mais l'entrée des scientifiques dans la commission de 1876, leur volonté proclamée d'une synergie entre le zoo et leurs institutions ne donnèrent que de faibles résultats. Les salles de physiologie et d'anatomie comparée ne furent jamais construites, et l'ouverture, en 1884, d'une nouvelle faculté des sciences, plus loin en aval du Rhône, entérina la séparation avec les laboratoires. La fusion avec le muséum, pourtant installé en bordure du parc en 1913, ne fut jamais importante: les Archives du Muséum d'histoire naturelle de Lyon, créées par Lortet en 1872, ne publièrent aucune étude sur le cheptel. Enfin, les scientifiques perdirent le contrôle du zoo en 1910, lorsqu'une véritable direction fut créée et confiée au vétérinaire municipal, inspecteur des abattoirs 42 . Il s'agissait désormais plus de pérenniser le troupeau que de l'étudier. Le transfert de la charge à un professeur de l'école vétérinaire de Lyon à partir de 1959 remit à l'honneur le dessein d'une collaboration scientifique 4, une intention reprise un peu partout en Europe au même moment. Mais un simple recensement des thèses

de Paris au maire, 22 novembre 1887.

40. Arch. mun. Lyon, 485 WP 4, Rapport vome, 23 décembre 1876; 485 WP 12, Rapport voirie, 17 mars 1874. Sur le constat général d'échec: Edouard TROUESSART [directeur de la ménagerie du Jardin des Plantes de Paris], «Les conditions, de l'hybridité en zoologie", dans Revue des idées, 1908, pp. 323-343; Edouard TROUESSART, «Acclimatation", dans Le Monde et la science, 1911, tome 1, pp. 15-28.

41. Arch. mun. Lyon, 485 WP 1, Programme donné à Bühler, 4 août 1856; Bernard MARREY et Jean-Pierre MONNET, La grande histoire des serres et des jardins d'hiver, France, 1780·1900, s.1., Édition Graphite, s.d.,p. 84.

42. Tête d'Or ..., ouv. cité, p. 31.

43. Voir le texte de Jean-Paul Deschanel, directeur du jardin zoologique depuis 1970, Ibidem, p. 31. 
vétérinaires soutenues à Lyon montre que de tels travaux sont peu nombreux, les étudiants (marché oblige) se spécialisant rarement dans l'étude des animaux exotiques. La direction du jardin est plus un élément de prestige et de pouvoir universitaires qu'une réalité scientifique.

Cette situation, quasi générale en Europe, résulte d'une fréquente inadéquation entre les intérêts de la science et les possibilités du zoo. Le parc aurait pu devenir un terrain de recherches en matière de systématique, c'est-àdire d'identification et de classification des espèces, mais celle-ci fut surtout à l'honneur dans les premières décennies du $\mathrm{XIX}^{\mathrm{e}}$ siècle, et elle se focalisa, sous l'impulsion de Cuvier, sur l'étude des caractères internes, donc sur un travail de dissection des dépouilles 44. De la même manière, la physiologie, très en pointe au milieu du siècle, puis les théories évolutionnistes, s'intéressèrent moins aux différences entre espèces qu'aux fonctions et aux évolutions des organes. Les quelques travaux qui eurent lieu au parc montrent par conséquent un usage particulier $\mathrm{d} \sim \mathrm{s}$ animaux, soit comme une réserve biologique disponible pour des expériences sur les maladies et leurs traitements, soit comme un stock d'attente avant les dissections post mortem pour la systématique, l'anatomie comparée ou les naturalisations du muséum 45. Ils n'utilisèrent pas le zoo comme un laboratoire particulier, conduisant des études spécifiques, qui justifieraient son existence. L'éthologie, constituée à partir des années 1930, aurait pu combler ce vide, mais elle s'est peu développée en France, à la différence des pays anglo-saxons, et les enclos souvent exigus des zoos ne permettent guère des études fiables. De leur côté, les travaux vétérinaires s'intéressent plus à la zoopsychologie et à la traumatologie des espèces; ils ont donc moins pour but de les connaître en elles-mêmes que de les garder en bonne santé physique et psychique dans les conditions particulières qui leur sont faites 46 .

Il reste que les scientifiques agirent dès les années 1880 pour diversifier la " collection)\} et leurs critères de choix révèlent une optique muséographique privilégiant, jusqu'à une époque récente, la rareté et la curiosité zoologique, donc le nombre des espèces au détriment d'une présentation en famille ou en clan 47. Par sa prétention à l'énumération, voire à une certaine exhaustivité

44. Voir: Henri DAUDIN, Les classeszoologiques et l'idée de série animale en France à l'époque de ,Lamarck et de Cuvier (1790.1830), Paris, Alcan, 1926.

45. Arch. mun. Lyon, 485 WP 4, Pré-rapport de la commission, 1876; 485 WP Il, Maire à école vétérinaire, 20 mai 1899.

46. Exemples: Michel JACQUIN, Contribution à l'étude de la contention des animaux sauvages, Thèse Maisons-Alfort, 1960; Jean-Marc LERNOULD, Contribution à l'étude de la traumatologie chez les animaux sauvages en captivité, Thèse MaisonsAlfort, 1968 ; Daniel PASCAL, Les girafulés en parc zoologique, Thèse, Lyon, 1971.

47. Arch. mun. Lyon, 485 WP 12, Procès verbal du conseil municipal, 25 janvier 1889. On trouve de nombreux exemples de cescritères dans: 485 WP 10 et 11. 
grace a une comp lementante , un temps rech $\mathrm{h}_{\mathrm{erc}} h_{\text {ee }}^{\prime \prime}$ avec $\mathrm{l}_{\mathrm{e}}$ museum ${ }^{48}$,cette collection servit à recenser et à connaître la nature, à affirmer la mainmise de l'homme moderne.

En fait, le véritable but du jardin zoologique, avoué dès 1856 par la municipalité, fut la distraction du public. Dès l'ouverture, le parc connut un succès qui ne se démentit guère ensuite. Il devint un lieu privilégié de promenade, marqué par une certaine différenciation sociale: plutôt la semaine pour les couches aisées, habitant le voisinage ou disposant de loisirs, plutôt le dimanche et les jours fériés pour les autres, spécialement les plus populaires 49. Mais ce fut surtout le jardin zoologique, et notamment les enclos «animés », qui intéressèrent: la commission de 1876 attribua directement la moindre fréquentation du parc dans les années 1870-1875 au déclin concomitant du cheptel, qu'elle considérait comme l'attrait le plus puissant, et elle déclara que sa reconstitution serait le moyen le plus efficace du renouveau; en 1881, on voulut attirer le public le long de la digue du Rhône, une zone délaissée, en y installant des chameaux, des zèbres, des yacks 50 .

Face au succès populaire, sans doute dû à la progressive généralisation du repos dominical et de sa promenade, l'idée que le jardin zoologique serait avant tout destiné aux classes laborieuses s'imposa assez vite parmi les administrateurs. En 1873, le gérant affirma que le parc était le jardin des pauvres, et que le zoo devait leur fournir d'honnêtes distractions. Au loisir succéda l'instruction dans l'Entre-deux-guerres, une époque d'intenses efforts de scolarisation primaire, où les élites économiques, syndicales, politiques, partageaient le sentiment que les loisirs populaires devaient être un temps privilégié d'éducation, bien choisi, bien encadré: le zoo devait donc donner de concrètes leçons d'histoire naturelle. C'est pourquoi la mode des jardins municipaux, agrémentés de quelques bêtes, se développa dans les villes moyennes. Mais, derrière ces convictions, se profile la distinction sociale étudiée par Pierre Bourdieu, réservant les activités de l'esprit - concert, théâtre, musée - aux élites, et privilégiant une culture plus concrète pour les couc hes popu lärres ${ }^{51}$.

48. Jules HUTINEL, Une visite aux animaux du parc de la Tête d'Or de Lyon, Lyon, Imprimerie nouvelle, 1908, pp. 44-45.

49. Catherine PELLISSIER, Loisirs et sociabilités des notables lyonnais au XIX' siècle, Tome 1, Lyon, Presses universitaires de Lyon/Éditions lyonnaises d'art et d'histoire, 1996, p. 231

50. Arch. mun. Lyon, 485 WP 4, Pré-rapport de la commission, 1876, Lortet au maire, 3 mai 1881.

51. ESTIENNE, Observations présentées à MM les membres de la commission municipale, Lyon, Bellon 1873; Arch. mun. Lyon, 963 WP 37, Muséum de Paris au maire, 2 février 1933; $961 \mathrm{WP}$ 72, Société des Amis du zoo de Strasbourg au maire, 7 octobre 1935. Les loisirs: Alain CORBIN [dir.], L'avènement des loisirs..., ouv. cité, pp. 140, 316, 389, et notamment Anne-Marie THIESSE, «Organisation des

Cahiers d'Histoire, nO 3-4, 1997, L'animal domestique, pp. 677-706 
Il reste que le public s'appropria le jardin zoologique à sa manière. Sans qu'il l'exprime d'une manière officielle - mais les administrateurs le reconnurent -, il fit rapidement le choix des animaux exotiques, rares et curieux, au détriment des bêtes domestiques ou d'acclimatation, d'abord privilégiées par la municipalité 52. Cette prédilection constitua le facteur fondamental de la transformation progressive du jardin, de la ferme vers le zoo exotique. Elle obligea, dès les années 1860, à introduire quelques animaux rares et curieux pour compenser la présence des bêtes domestiques qui n'attiraient guère; elle fut à la base du déclin de fréquentation des années 1870; elle conforta la préférence des scientifiques envers les bêtes exotiques après 1881, car elle s'alliait à leur désir de collection; elle devint le seul critère de choix de la municipalité après 1918, et elle la poussa à transformer le mode de présentation des animaux dans les années 1960.

Cette faveur fut d'abord celle des enfants, dont l'importance croissait dans les familles, qui décidaient des trajets, imposaient les arr\&ts, et créaient le succès public de telle ou telle bête. Car, et cela n'a pas changé, l'intérêt se

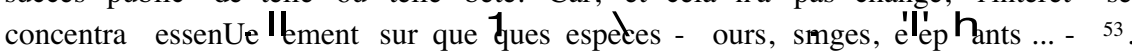
Il révèle une vision de l'animal différente de celles du zootechnicien, du scientifique ou du vétérinaire, fondée sur le sentiment de proximité, le jeu, la facétie. Il s'inscrit dans le contexte général de la passion pour l'exotisme, qui étreignit l'Occident à partir du romantisme, qui fut renforcée par l'aventure coloniale et qui poussa de plus en plus, d'abord les élites, puis les classes moyennes au XX $X^{\mathrm{e}}$ siècle, à voyager à l'étranger. En ce sens, le zoo fut pour beaucoup, et jusqu'aux années 1960, avant le tourisme de masse, un succédané des vacances et des voyages. L'intérêt pour les espèces spectaculaires s'inscrit aussi dans un contexte local de développement des attractions durant la seconde moitié du $\mathrm{XIX}^{\mathrm{e}}$ siècle, la municipalité installant au parc ce qu'elle ne savait où mettre: des activités sportives - cricket, patinage à glace ... répondant à la demande des élites libérales du voisinage, attirées par les nouveaux sports, mais surtout des attractions festives - boules, promenades à vélocipèdes, concerts, théâtre de guignol, canotage ... -, révélatrice du développement d'une sociabilité foraine sous la Troisième République. D'ailleurs, des cafés-concerts et des cirques s'installèrent dans le quartier 54.

loisirs des travailleurs et temps dérobés (1880-1930)", pp. 302-322; Pierre BOURDIJ;:U, La distinction, critique sociale du jugement, Paris, Editions de Minuit, 1979; Catherine PELLISSIER, Loisirs et sociabilités ..., ouv. cité, pp. 183-207.

52. Arch. mun. Lyon, 485 WP 10, Rapport voirie, 17 septembre 1881 j 485 WP 11 ,

M. Petit au maire, 27 mai 1897 j 961 WP 72, Directeur zoo au maire, 23 janvier 1923 et 4 octobre 1929; Jules HUTINEL, Une visite aux animaux ..., ouv. cité, p. 36 et p. 39.

53. Cet intérêt pour les espèces "spectaculaires" se retrouve partout: Emilio SANNA, Cet animal estfou, Paris, Librairie Arthème Fayard, 1976, pp. 176-177.

54. On trouvera en Arch. mun. Lyon, 485 WP 10, un dossier de concessions d'activités. 
Le public s'appropria aussi le jardin, en établissant des relations particulières avec les bêtes. Comme dans tous les zoos, il prit rapidement l'habitude de les alimenter. L'administration s'y opposa sans cesse au regard des problèmes engendrés (gavage, obésité, mortalité). Mais cette rationalité est restée vaine, jusqu'à nos jours, face à la fonction symbolique du don d'aliments, souvent soulignée par les ethnologues et les éthologistes : il permet le contact, agit comme un signe de paix - il s'accompagne souvent d'une caresse -, signifie le désir d'un échange 55. Cependant, dans le cas du zoo, il peut aussi traduire une volonté plus ou moins consciente et exprimée de nier le sauvage, de le faire entrer dans la sphère de la civilisation. Cela expliquerait certains accidents, assez fréquents dans l'histoire des zoos, tel celui de 1887 à Lyon, où un homme escalada une grille pour donner à manger à un ours qui ... lui mordit la main et l'envoya quatre fois en l'air! 56

Un écart entre une partie du public et les élites dans les usages du jardin se maintint jusqu'aux années 1950, car certains, mal identifiés par les textes, ne le considérèrent pas comme une école populaire mais comme un garde-manger dans lequel on pouvait choisir divers volatiles, une tendance encore plus sensible pendant les guerres, et même présente chez quelques gardiens indélicats 57. La violence, celle du temps vis-à-vis des bêtes, fit aussi son entrée au jardin, dès les origines, et se concrétisa par des oiseaux décapités, des singes empoisonnés, etc. Elle s'inscrivait dans un usage populaire du parcfraudes diverses, contrebande, débauches sexuelles -, que l'administration élimina peu à peu. Elle-même, patiemment réprimée par les élites, à l'échelle nationale, pour obtenir un façonnement des mœurs et des corps, semble $\mathrm{s}$, $\mathrm{ff}_{\mathrm{acer}}$ au zoo apre's ID" euxleme guerre mon $\mathrm{d}^{\prime} \mathrm{al}_{\mathrm{e}}{ }^{58}$.

Sur la recherche de l'exotisme et la sociabilité citadine du temps: Alain CORBIN [dir.], L'avènement des loisirs..., ouv. cité, pp. 98-100, et notamment: Julia SCERGO, «Extension et mutation du loisir citadin, Paris $\mathrm{XIX}^{\mathrm{e}}$ siècle-début $\mathrm{XX}^{\mathrm{e}}$ siècle ", pp. 145-165; Catherine PELLISSIER, Loisirs et sociabilités ..., ouv. cité, pp. 224, 229 ; Germaine VIEUX, «Théâtres, bals et cirques ... sur la rive gauche ", dans Rive Gauche, nO 35-39, décembre 1970-décembre 1971.

55. Jules HUTINEL, Une visite aux animaux ..., ouv. cité, p. 20; Arch. mun. Lyon, 877 WP, liasse 272, Directeur au maire, 18 juin 1965; «Le parc de la T\&te d'Or '>, dans Lyon Horticole, 1971, p.39. Sur le symbolisme du don: Denis BUICAN, Ethologie comparée, Paris, Hachette, 1995, p. 116; Jane VAN LA WICK-GOODAL, Les chimpanzés et moi, Paris, Éditions Stock, 1991, qui commença ainsi son étude au parc de Gombe.

56. Arch. mun. Lyon, 485 WP 12, Préfet au maire, 10 septembre 1887.

57. Arch. mun. Lyon, $485 \mathrm{WP}$ 14, Estienne à préfet, 8 septembre 1873; 961 WP 73, Directeur zoo au maire, 22 novembre 1926; 963 WP 37, Correspondance Président délégation spéciale-directeur zoo, 11 et 28 avril 1941 ; 485 WP 4, Voirie au maire, 3 mars 1903.

58. Arch. mun. Lyon, 485 WP 10, Voirie au sénateur, 30 mai 1860; 961 WP 73, Directeur zoo au maire, 27 juillet 1925 et 2 mars 1928. On trouvera dans 485 WP 4 
Un autre phénomène peut confirmer qu'il s'agit bien d'une réalité, d'une pacification des esprits, signe d'une évolution des mentalités, et non d'un silence des archives. C'est la montée parallèle d'un courant d'opposition aux conditions de vie des animaux. Il était déjà présent en filigrane, vers 1880-1900, dans la volonté de Lortet d'améliorer les installations pour ne pas mécontenter les spectateurs ou dans les exigences de confort émises par certains donateurs 59. Il devint public dans l'Entre-deux-guerres, avec des critiques orales remontant jusqu'aux oreilles du directeur, des lettres de protestation individuelles ou collectives, d'« un groupe d'habitués du parc ", d'« une amie des bêtes et ses amis », de la direction de la SPA de Paris alertée par ses sociétaires lyonnais, à propos de l'exiguïté ou de la malpropreté des cages, de sols boueux, de l'état lamentable d'une bête, de l'attitude brutale des gardiens, etc. 60.

Bien qu'ils fussent les reflets d'une sensibilité croissante au sort de l'animal et d'une montée du niveau de vie, qui rendaient intolérables des conditions jugées normales 50 ans plus tôt, ces reproches ne signifièrent pas une opposition au principe même du jardin zoologique, bien au contraire. En 1908, Jules Hutinel, secrétaire honoraire de l'inspection académique de Lyon, publia Une visite aux animaux du parc de la Tête d'Or à la demande de la SPA de Lyon et du sud-est, afin d'informer le public sur le physique, l'instinct, les mœurs des animaux. Jusqu'aux années 1980, ladite société, fidèle à la conception éducative des zoos évoquée plus haut, utilisa le parc comme un lieu d'instruction populaire et d'apprentissage au respect de l'animal. Elle organisa des visites et sollicita la collaboration du directeur pour présenter dans son bulletin la grande rénovation des décennies 1960-1970 . À l'inverse, les idées protectrices trouvèrent plus qu'un écho favorable auprès des directeurs: le docteur Didier, vétérinaire municipal, directeur du zoo de 1910 à 1938, fut l'un de ses membres. Lui et Lortet utilisèrent les critiques pour tenter d'obtenir des

un dossier sur les délits au parc. Sur la violence du XIX ${ }^{\mathrm{e}}$ siècle envers l'animal : Maurice AGULHON, «Le sang des bêtes: le problème de la protection des animaux en France au $\mathrm{XIX}^{\mathrm{e}}$ siècle ", dans Sangs.- Romantisme, revue du XIX' siècle, $\mathrm{n}^{\circ}$ 31, 1981, pp. 81-109, réédité dans Maurice AGULHON, Histoire vagabonde. J. Ethnologie et politique dans la France contemporaine, Bibliothèque des histoires, NRF, Paris, Gallimard, 1988, pp. 243-282; Éric BARATAY, «Représentations et métamorphoses de la violence: la corrida en France (1853 à nos jours) ", dans Revue Historique, 1997, nO 2, pp. 489-520.

59. Arch. muni Lyon, 485 WP 11, Lortet au maire, 3 mai 1881; Directeur Havas-Lyon au maire, 14 avril 1895 ; M. Petit au maire, 27 mai 1897.

60. Arch. mun. Lyon, 963 WP 37, Directeur zoo au maire, 4 novembre 1926 et 10 mars 1939; particuliers au maire, 3 juin 1938; 961 WP 72, Directeur zoo au maire, 4 octobre 1929; lettres de particuliers au maire, 20 avril 1930, 3 juin 1930, 30 octobre 1930,23 mars 1932.

\%61. Jules HUTINEL, Une visite aux animaux ..., ouv. cité, p.3; La protection des animaux. Revue de la Société protectrice des animaux de Lyon et du Sud-Est, nO 45, 1970 , p. 15 , nO 46,1970, pp. 8-9, nO 58, 1973, p. 22, nO 66, 1975, pp. 17-18. 
améliorations dans les installations. Cette proximité doit rappeler que, depuis sa fondation, l'objectif de la SPA n'était pas de remettre en cause le principe de la domination de l'homme sur le monde animal, mais d'en combattre les abus, et qu'elle eut longtemps une moindre attention à l'animal sauvage, et encore plus exotique, par rapport à l'animal domestique et à certains aspects mauvais traitements, vivisection, corrida, abandons - jugés plus importants. Elle partagea d'ailleurs, au XIX ${ }^{\mathrm{c}}$ siècle, l'ambition d'une acclimatation et d'une domestication de la faune sauvage 62 .

Ce ne fut donc que dans les décennies 1960-1970 qu'apparut, en France et en Europe, une contestation du principe même des zoos, considérés comme inutiles à l'heure de la télévision et des voyages, inadaptés aux

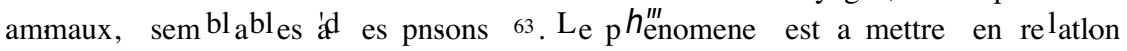
avec le contexte de la décolonisation, du tiers-mondisme, et du procès de l'Occident, mais aussi avec la remise en cause des formes d'aliénation dans la société contemporaine. Nombreux furent alors ceux qui comparèrent les prisons, les hôpitaux psychiatriques et les zoos 64. Cette contestation ne fut pas générale et provoqua des tiraillements parmi les protecteurs. Le cas lyonnais montre que la SPA locale ne fut pas à la pointe mais à la traîne des critiques, qui furent d'abord individuelles. Après une brève parenthèse en 1974, elle ne leur ouvrit vraiment les colonnes de son bulletin qu'au début des années 1980 et, bien qu'elle réduisît peu à peu l'utilisation pédagogique du zoo, elle resta prudente, donnant autant de place à ses partisans, préférant faire un tri entre les bons et les mauvais établissements, laissant les condamnations de principe à des associations plus récentes et plus radicales, telle la Ligue française des droits de l'animal 65. Cependant, même à l'échelle nationale, le zoo demeure le parent

62. Sur les objectifs de la SPA, voir: Michel FLEURY, La belle histoire de la SPA, Paris, Grasset, 1995. Sur l'adhésion à la domestication et l'acclimatation: Claude BLANCKAERT, «Les animaux "utiles" ... ", art. cité. La SPA de Lyon s'était "agrégée" à la Société d'acclimatation en 1861: Bulletin de la Société impériale d'acclimatation, $1861, \mathrm{p} . \mathrm{V}$.

63. Un exemple dans: «Le parc de la Tête d'Or », dans Lyon Horticole, 1er trimestre 1971, pp. 35-36. Pour la contestation des zoos, l'ouvrage le plus important en France, par l'écho qu'il déclencha, fut celui de: Philippe DIOLE, Les animaux malades de l'homme, Paris, Flammarion, 1974.

64. Voir l'exemple du psychiatre canadien: H. HELLENBERGER, «Jardin zoologique' et hôpital psychiatrique », dans Abel BRION, Henri HEY [dir.], Psychiatrie animale, Paris, Desclée/De Brouwer, 1964, pp. 559-578, et celui du journaliste italien, spécialiste des prisons: Emilio SANNA, Cet animal est fou ..., ouv. cité. En France, si des protecteurs firent de même, la contestation des hôpitaux et des prisons ne s'étendit pas aux zoos 'exemple de Michel Foucault est symbolique), signe supplémentaire d'une spécificité française. Sur celle-ci, voir: Éric BARAT AY, « Représentations et métamorphoses de la violence ... ", art. cité.

65. La protection des animaux, ouv. cité, nO 63, 1974, pp. 9-13, nO 93, 1982, p. 23, nO 97, 1983 , p. 14 , nO 106, 1985, pp. 14-15; Georges CHAPOUTIER et Jean-Claude 
pauvre de la protection, sans doute sous le poids de l'idéologie d'origine évoquée plus haut.

\section{UNE MISE EN SCÈNE DES ANIMAUX}

La mise en spectacle des animaux répond au désir de voir de la part du public. Elle révèle la fonction essentielle $\mathrm{du}$ jardin zoologique, mais elle indique aussi les conceptions ambiantes de la nature et les desseins que l'homme nourrit à son égard. Les premières installations, d'abord en 1859-1861 puis dans les décennies 1870-1880, furent des parcs - aux vaches, moutons, daims, volailles, etc. - avec des abris, des cabanes et divers bâtiments faisanderie, colombier, vacherie, volière -, le plus souvent en bois, inspirés des fermes ou des chalets montagnards aux toits élancés 66. L'ensemble satisfaisait l'envie de pittoresque, la volonté de créer une nature tantôt sauvage, tantôt domestiquée, animée par des troupeaux, le goût d'une ethnographie plus ou moins folklorique à l'époque de l'inventaire du monde, mais aussi l'ambition, et sans que cela parût contradictoire, d'intégrer toute la nature dans le cadre européen pour la mettre au service de l'homme.

L'arrivée, dans un deuxième temps, d'animaux plus sauvages, plus dangereux ou jugés plus exotiques entraîna l'édification de bâtiments plus élaborés, qui donnèrent vraiment au parc un air de jardin zoologique: cages aux ours en 1865 et 1882, aux singes en 1880, pour des crocodiles en 1894, des fauves en 1922 et un éléphant en 1924. À chaque fois, on entreprit d'abord d'installer les bêtes aux moindres frais, en essayant tous les palliatifs: tentative de division en trois parties de la cage aux ours en 1882; hébergement de deux crocodiles dans la serre des plantes aquatiques, puis construction d'un petit abri adjacent Oes excréments faisaient périr les plantes), qui devint trop réduit en 1894 ; intention de placer deux lions dans la maison des crocodiles, la cage aux ours ou la volière en 1917 ! Le coût des travaux fit abandonner plusieurs projets, et les autres ne furent souvent réalisés que dans l'urgence, avec un souci d'économie à courte vue: on transforma la vacherie, construite par Tony Garnier en 1904-1906, en fauverie et en maison de l'éléphant, mais celleci, trop légère et exiguë, dut être revue dès... 1927! Chaque construction donna lieu à des tractations et à de mauvais compromis entre une municipalité préoccupée de limiter les budgets, des architectes soucieux d'affirmer leur autonomie face aux contingences matérielles tout en étant contraints de réduire leurs projets, et un directeur plaidant pour la rénovation, reprenant souvent à son compte les critiques du public, mais sans vraiment pouvoir imposer ses vues ${ }^{67}$.

NOUËT [dir.], Les droits de l'animal aujourd'hui, Paris, Arléa/Ligue française des $\sim$ droits de l'animal, 1997.

66. On trouvera des devis et descriptions dans: Arch. mun. Lyon, 485 WP 2 et 13.

67. On trouvera les dossiers de construction, avec correspondance, devis, plans, dans: 
Le résultat fut la construction de bâtiments jamais adaptés aux animaux, avec notamment des superficies réduites - d'après les plans: à peu près $3 \mathrm{~m}^{2}$ pour la tanière des ours et $50 \mathrm{~m}^{2}$ pour leur cage, autant pour la maison des crocodiles, de 13 à $22 \mathrm{~m}^{2}$ en cages internes et de 20 à $35 \mathrm{~m}^{2}$ en cages externes pour les fauves, 53 et $50 \mathrm{~m}^{2}$ environ pour l'éléphant - ne permettant pas une vie physiologique et psychologique normale, un défaut reconnu plus tard, mais qui étaient proches des normes urbaines de l'époque et qui apparurent donc décentes: en 1896, $71 \%$ des logements lyonnais étaient composés de une à trois pièces estimées à $10 \mathrm{~m}^{2}$ en moyenne, soit une superficie de 10 à $30 \mathrm{~m}^{2}$, et $55 \%$ seulement avaient une concession d'eau 68. En prenant ainsi l'univers humain pour référence, on déniait toute spécificité au monde sauvage, qu'on voulait, plus ou moins inconsciemment, intégrer dans la civilisation.

L'autre problème résidait en une humidité chronique du parc, une aération insuffisante, et un chauffage déficient des tanières. Le poêle installé dans la singerie, dans les années 1880, dégageait plus d'acide carbonique que de chaleur, et celui placé dans la fauverie en 1923 fonctionnait pour $231 \mathrm{~m} 2$ !69 D'où une forte mortalité annuelle, reconnue par la direction, avec des pointes l'hiver, par suite de congestions pulmonaires, de pneumonies, d'angines, de problèmes gastriques, etc. Il n'existe pas de statistiques continues, néanmoins des données éparses montrent qu'elle pouvait être importante: 17,3\% en $1876,43,5 \%$ en 1878, affectant alors surtout des volatiles, mais aussi des singes pendant l'hiver 1900 ou de gros mammifères (éléphant, ours) durant celui de $1940^{70}$. À cet état chronique s'ajoutait une mortalité rapide à l'arrivée au zoo, en raison des conditions de transport - longs voyages en des caisses exiguës ou lors des deux guerres mondiales du fait des restrictions 71 . Il ne s'agissait pas

Arch. mun. Lyon, $485 \mathrm{WP} 10$ pour les ours et les crocodiles, $961 \mathrm{WP} 73$ pour les fauves, 963 WP 37 pour l'éléphant. Sur les demandes des directeurs: 485 WP 4, Lortet au maire, 3 mai 1881; $961 \mathrm{WP} 72$, Rapport au maire, 7 septembre 1928; 963 WP 37, idem, 15 juin 1938.

68. Gustave LOISEL, Histoire des ménageries..., ouv. cité, tome 3, p. 115, trouva les installations belles. Critiques postérieures dans: Arch. mun. Lyon, $925 \mathrm{WP}$ liasse 575/2, Rapport d'architecte sur la fauverie, 1973. Les logements lyonnais: Jean-Luc PINOL, Mobilités et immobilisme d'une grande ville ..., ouv. cité, pp. 266273.

69. Arch. mun. 1.yon, $485 \mathrm{WP}$ 10, Maire à voirie, 8 octobre 1886; 963 WP 37, Plan de la fauverie, 1923.

70. Arch. mun. Lyon, 485 WP 4, Rapport voirie, 23 décembre 1876; 485 WP 12, Situation au premier octobre 1879, et État des changements, 1900; 963 WP 37, Directeur au maire, 27 avril 1940.

71. Arch. mun. Lyon, 485 WP 11, Rapport voirie, 27 juin 1888 (castors) ; 961 WP 72, Directeur au maire, 24 septembre 1928 (panthère); $963 \mathrm{WP} 37$, Directeur au président de la délégation spéciale, 10 juin 1941 et Rapport au préfet, 1erjuillet 1942.

Cahiers d'Histoire, nO 3-4, 1997, L'animal domestique, pp. 677-706 
d'une négligence de la part de la direction. Les caractéristiques de l'alimentation montrent qu'elle désirait garder son cheptel: viande de cheval de bonne qualité pour les carnassiers, alors que les autres ménageries utilisaient souvent des viandes saisies aux abattoirs; avoine, foin, son, pommes de terre et pain de premier choix ou sans moisissures ni altérations pour les autres 72 . Il s'agissait plutôt d'une incompréhension générale vis-à-vis du monde sauvage, et d'une volonté de le présenter en fonction de critères humains comme nous allons le voir. D'ailleurs, les pertes étaient largement compensées par les reproductions - essentiellement des volatiles, mais aussi des cervidés et quelques mammifères dans l'Entre-deux-guerres - et surtout par les multiples dons des coloniaux et des particuliers 73. Là aussi, le parc participa, comme tous les jardins zoologiques, à l'entreprise occidentale de mainmise sur la nature exotique, qui se traduisit par une destruction massive de la faune. Le phénomène fut tel qu'il inquiéta les gouvernements anglais et allemand au début du $\mathrm{XX}^{\mathrm{e}}$ siècle, et susclta $\mathrm{I}_{\text {es premIeres mesures }} \mathrm{d}_{\mathrm{e} \text { protectlOn }}{ }^{74}$.

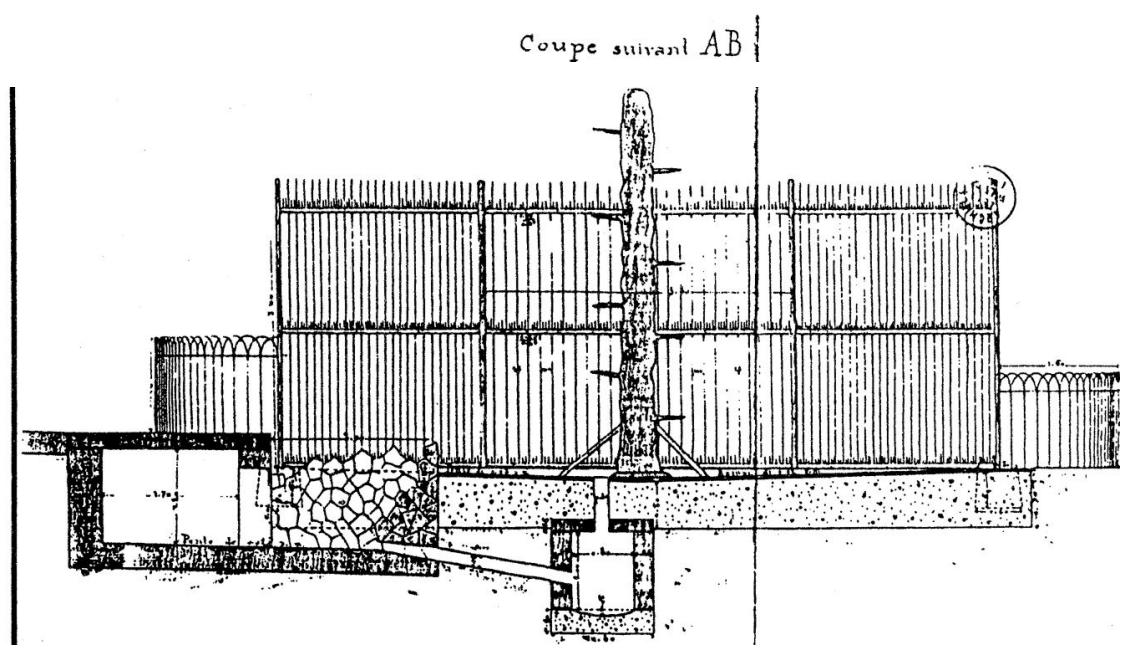

Figure 1: Plan d'une fosse aux ours, 1877 (Arch. mtln. Lyon, 485WP10)

72. Arch. mun. Lyon, 485 WP 12, Marchés pour la fourniture des denrées, 1883, 1887 ; 961 WP 37, Directeur au maire, 12 novembre 1930.

73. États de mouvements d'effectifs en: Arch. mun. Lyon, 485 WP 12, État des changements pour 1900; 963 WP 37, Directeur au maire, 15 juin 1938, qui évoque la reproduction des fauves.

74. Voir: H. CONWENTZ, The Care ofNatural Monuments with Special Reference to Great Britain and Germany, Cambridge, Cambridge University Press, 1909. 
Ainsi les bâtiments (exemple figure 1) répondaient à une zoologie sommaire: quelques mètres carrés pour des animaux finalement réduits aux fonctions végétatives - manger, boire, évacuer, dormir -, estimés sans lien avec leur milieu naturel, assimilés à des objets - les documents de transport parlent sans cesse de «collection» ou de «matériel» «emballés "; le directeur considéra, en 1938, la panthère comme un «animal d'ornement), 75 - ; quelques éléments jugés indispensables aux fonctions biologiques vitales 76 ou caractéristiques de leur cadre d'origine - tanière, bassin, rocher et tronc d'arbre pour les ours, branches pour les singes, bassin et plage pour les crocodiles, tanières pour les fauves, bassin pour l'éléphant -, mais qui le symbolisaient plus qu'ils ne le reproduisaient, qui fonctionnaient plutôt à destination du public pour qu'il l'imagine. D'où la mode, un peu partout en Europe, du style ethnographique, comme par exemple, à Lyon, ce pavillon de style mauresque pour abriter les crocodiles du Nil !

En fait, les installations garantissaient avant tout trois besoins humains. D'abord que le public puisse voir. On créa pour cela une véritable scénographie: les cages aux ours, les premières de la série, furent placées en un point de convergence de plusieurs allées, et une sensation de gigantisme de l'animal fut générée par la vue préalable de vastes pelouses et d'animaux de petites tailles 77. Par la suite, les autres bâtiments furent, non pas dispersés, mais rassemblés pour donner l'impression d'une zone africaine. A cela s'ajoutèrent la théâtralisation des cages, que nous venons de voir, et une recherche de transparence: les formes hexagonales ou circulaires des enclos externes devaient permettre au plus grand nombre de regarder de tous les côtés, quitte à ce que les bêtes subissent les courants d'air.

Une autre exigence, à laquelle on fit attention dès les premières constructions, concerna l'hygiène: sol en pierre ou en ciment - interdisant de creuser -, pentes régulières, canalisations, égout central, devaient faciliter l'évacuation des excréments, des urines, des eaux usées. Cela répondait à la volonté du personnel de travailler aisément, mais aussi à une aversion croissante pour les miasmes à l'heure de l'affinement progressif des odorats 78 .

La dernière nécessité fut celle de la sécurité des gardiens et du public. Elle constitua une véritable hantise pour la municipalité, qui craignait de voir

75. Arch. mun. Lyon, 961 WP 73, Directeur à maire, 22 septembre 1934; 963 WP 37, idem, 5 décembre 1938.

76. Voir les critères d'installation des ours, des castors ou des phoques en: Arch. mun. Lyon, 485 WP 10, Rapport voirie, 18 octobre 1877; 485 WP 11, Jardin d'acclimatation de Boulogne au maire, 4 juin 1886 ; 961 WP 72, Directeur au maire, 18 avril 1935.

77. Arch. mun. Lyon, 485 WP 10, Plan général, 22 aOl'it 1894.

78. Arch. mun. Lyon, 485 WP 10, Plan de la fosse aux ours, 1877 ; 963 WP 37, Plan de la fauverie, 1923. Voir: Alain CORBIN, Le miasme et la jonquille. L'odorat et l'imaginaire social, XVIIfXIX' siècles, Paris, Aubier, 1982. 
sa responsabilité engagée dans un accident. Elle permit d'ailleurs au directeur d'obtenir des rénovations inaccessibles autrement. En 1926, il signala que l'exiguïté de la cage externe de l'éléphant et l'absence d'un bassin d'eau courante faisaient l'objet de vives critiques de la part des spectateurs et n'assuraient pas une vie active au pachyderme. Mais il avança surtout que cela le rendait enragé et qu'il risquait d'abattre les cloisons et les grilles en grandissant. Dès 1927, on renforça les murs, on construisit un bassin d'eau courante et un enclos de $245 \mathrm{~m}^{2}$ élimité par de solides barreaux de $35 \mathrm{~mm}$ de diamètre 79

Tous les projets, tous les plans, montrent une véritable obsession des barreaux, dessinés avec soin, calculés, testés, et des barrières, toujours doublées, voire triplées. Les architectures reflètent une peur des animaux et de la nature sauvages, considérés comme cruels, féroces, un sentiment aux origines lointaines, véhiculé en particulier par le christianisme, repris par les philosophes modernes, en grande partie pour justifier la mainmise de l'homme, et largement présent dans les récits de chasses exotiques, très populaires jusqu'aux années 1950, qui dressèrent les carnivores et les grands

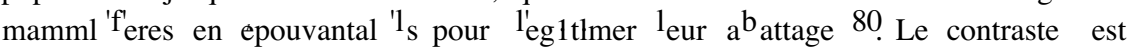
frappant au parc entre le traitement réservé aux animaux «paisibles» Oes herbivores), en liberté surveillée dans leurs enclos, et celui des bêtes «féroces », placées dans des quartiers de sécurité.

Mais les bâtiments affirmaient en même temps la volonté de dompter et de vaincre cette férocité présumée, pour assurer la victoire de la civilisation sur la nature. L'idée est présente dans le dessin de la fosse aux ours (figure 1) et encore plus dans ce projet de cage aux lions de 1917 (figure 2), non réalisé pour une simple raison budgétaire 81: il prévoyait une cascade de rochers dans la cage externe pour rappeler les chaînes de l'Atlas, mais cet élément de nature devait être enserré entre la culture - une architecture toute classique - et la force -les grilles. En donnant une impression d'inventaire et de collection, la présentation dans des cages parallèles, pour les singes ou les fauves, renforçait cette volonté de maîtrise.

79. Arch. mun. Lyon, 963 WP 37, Directeur au maire, 6 mai 1926, 4 novembre 1926, 30 avril 1927 ; Architecte au maire, 25 juin 1927.

80. Voir: Éric BARAT AY, L'Eglise et l'animal (France, XVIf- $X X^{\prime}$ siècle), Paris, Cerf, 1996 ; Stéphane BOULC'H, "Le statut de l'animal carnivore et la notion de pureté dans les prescriptions alimentaires du haut Moyen Âge chrétien", dans Liliane BODSON [dir.], Le statut éthique de l'animal,' conceptions anciennes et nouvelles, Liège, Université de Liège, 1996, pp. 41-59. Un exemple édifiant de récit de chasse avec: LE VAILLANT, Voyage de ML V.dans l'intérieur de l'Afrique par le cap de Bonne Espérance, Paris, Leroy, 1790.

81. Arch. mun. Lyon, $961 \mathrm{WP} 73$, Architecte au maire, 12 juillet 1917, réponse le 16 juillet 1917. 
Le troisième temps de la mise en scène n'intervint pas dans les quelques rénovations des années 1930-1950 - pavillons pour ruminants-, mais dans celles des décennies 1960-1970, qui changèrent l'aspect du jardin zoologique. Elles résultèrent de la montée des critiques, assimilant de plus en plus le zoo à une prison, de leur prise en compte, voire de leur acceptation par

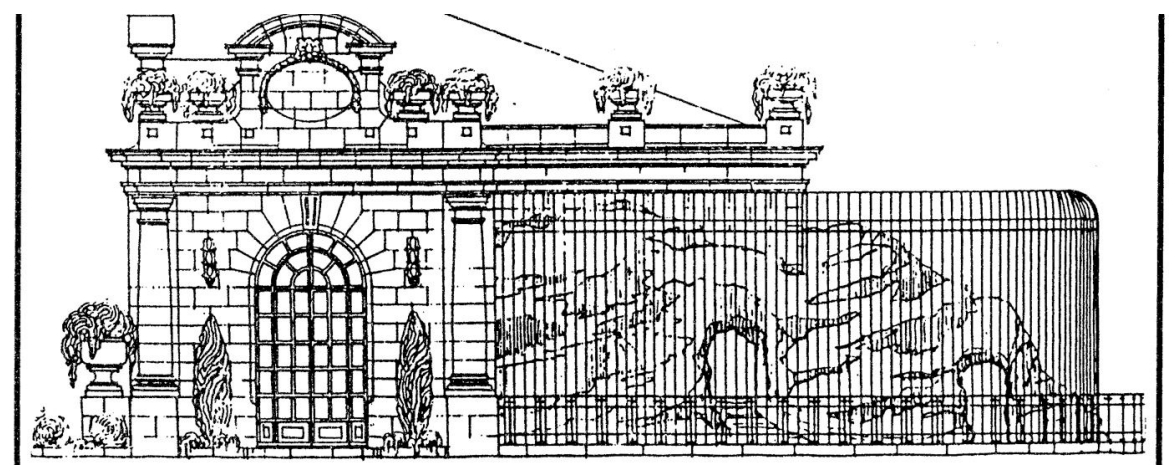

$\mathrm{EE} \sim \sim \mathrm{CIE} \sim \mathrm{COE} \quad \mathrm{A} \sim \sim \mathrm{J}_{-} \mathrm{J}<9 \mathrm{~J} \cdot \mathrm{J} \sim$

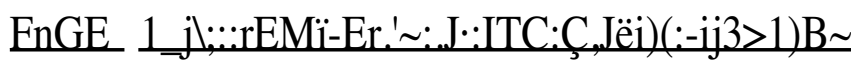

EGTiELUL $002 . M$

Figure II: Projet de cage aux lions, 1917 (Arch. mun. Lyon, 961WP73)

la municipalité, et de la volonté de Louis Pradel, maire de 1957 à 1976, de réagir contre l'inertie de la gestion d'Edouard Herriot dans les années 19301950, de moderniser la ville et de la rapprocher des métropoles européennes par une série de réalisations - réseau autoroutier, centre commercial et d'affaires, bibI'iothèque, musée, équipements sportifs et communaux - dont faisait partie la rénovation du parc - création d'une roseraie - et du jardin zoologique 82. Afin de rendre ce dernier digne de la réputation souhaitée de la ville, il reprit à son compte le projet du directeur de l'établissement de 1959 à

82. Françoise BAYARD et Pierre CAYEZ [dir.], Histoire de Lyon ..., ouv. cité, pp. 364, 435.

Cahiers d'Histoire, nO 3-4, 1997, L'animal domestique, pp. 677-706 
1967, le professeur Ferney de l'école vétérinaire de Lyon, qui proposait de le redessiner sur le modèle des grands zoos internationaux, en adoptant le principe de la semi-liberté inventé par le marchand d'animaux exotiques Carl Hagenbeck dans son jardin de Stellingen, près de Hambourg, en 1903-1907, et qui s'était diffusé dans toute l'Europe, en particulier au zoo de Vincennes, inauguré en 1934, qui servit de référence directe 83.

Par suite des contraintes budgétaires, d'autant plus lourdes, voire paralysantes que le parc ne disposait d'aucun fond propre, les réalisations s'étalèrent sur une trentaine d'années, un peu au hasard des crédits, des circonstances et de la volonté des maires successifs, Michel Noir (1989-1995) relançant un chantier arrêté après la mort de Louis Pradel: enclos des daims (1962), îles des oiseaux aquatiques, singerie (1964), maison des éléphants (1965), giraferie (1968), enclos des ruminants (1972), fauverie (1975), enclos des servals (1988), ourserie (1994).

Le premier principe d'aménagement fut la naturalisation des enclos extérieurs: terrains sablonneux ou herbeux, bassins, îlots avec plages, rochers, végétations et troncs d'arbres, etc. La combinaison de ces éléments devait permettre de créer des sites naturels. Le second principe fut celui de la semiliberté. Les barreaux et les grillages furent supprimés au maximum, souvent limités aux c8tés, et recouverts de végétation en cas de cages successives, remplacés le reste du temps par des fossés empêchant l'animal de sauter et de s'échapper. Les bêtes pouvaient ainsi évoluer dans des enclos élargis, situés à même hauteur que le public, ouverts sur l'environnement. Il s'agissait finalement d'étendre à la plupart des animaux et d'améliorer l'idée originelle duJär $\mathrm{d}_{\text {In amine }}^{\prime}$

La naturalisation se traduisit par la création d'enclos ensoleillés, plus vastes - $2000 \mathrm{ml}$ pour les éléphants, autour de $100 \mathrm{ml}$ pour certains fauves, soit cinq à dix fois plus que les précédents -, et s'accompagna d'une amélioration des locaux internes, mieux aérés, mieux chauffés - chauffage central, au sol, à air pulsé - et disposant de salles de soin de manière à réduire les pertes. Ces transformations peuvent s'analyser comme une résultante des Trente glorieuses, de l'amélioration du niveau de vie, et notamment de l'habitat, encore une fois plaqué sur le zoo. La volonté de remplacer les individus isolés par au moins un couple ou plusieurs éléments, et de favoriser la reproduction -les nouveaux bâtiments comprennent des salles d'isolement pour les naissances - s'inscrivit aussi dans cette recherche de nature 85.

83. Arch. mun. Lyon, 849 WP liasse 434, Directeur au maire, 17 octobre 1967; Carl HAGENBECK, Cagessans barreaux, Paris, Nouvelles Éditions de Paris, 1951.

84. Sur cet aspect et ce qui suit, voir les dossiers, avec plans, devis et correspondance, dans: Arch. mun. Lyon, 877 WP liasse 272 (éléphants), 849 WP liasse 434 (giraferie), $852 \mathrm{WP}$ liasses 575, 576 (fauverie), et le plan général de 1964 dans 877 WP liasse 271.

85. Voir les plans cités ci-dessus et en 852 WP liasse 575/2, le rapport de l'architecte sur 
Mais tout cela resta en grande partie du domaine de l'artifice et de la mise en scène. La naturalisation ne fut pas totalement respectée - la singerie. perpétue le modèle ancien, l'enclos des éléphants est dénudé, le grillage est resté de mise pour les fauves les plus agiles, l'extérieur de l'ourserie est cimenté -, et elle n'était pas suffisante pour assurer une vie «normale », comme en témoigne la persistance des comportements stéréotypés dandinements des éléphants, promenades circulaires des fauves ... -, présents dans tous les zoos, révélateurs d'un ennui chronique, d'une absence d'activités physiques et psychiques. D'ailleurs, les. éléments utilisés - bassin, tronc d'arbre, sol meuble, enclos plus vaste, zone d'isolement, etc. - répondaient avant tout aux critères de la zoopsychologie mise en place par Heini Hediger 86, directeur des zoos de Bâle puis de Zurich, à partir des années 1940. Son but n'est pas de créer un environnement proche du milieu d'origine, chose impossible dans les zoos classiques par manque d'espace, mais de respecter les distances de fuite des espèces vis-à-vis des hommes pour délimiter les superficies des enclos, et d'utiliser quelques éléments repères tirés du milieu originel et jugés indispensables à l'équilibre psychologique de l'animal pour l'aider à prendre possession de son parc, à l'accepter comme sien, donc à faciliter la détention en éliminant une partie du stress.

Par ailleurs, la naturalisation ne concerna pas les cages internes marquées par des superficies stables - $30 \mathrm{~m}^{2}$ environ pour chaque éléphant, ce qui obligea au maintien des chaînes, $72 \mathrm{~m}^{2}$ pour les girafes, de 20 à $40 \mathrm{~m}^{2}$ pour les fauves -, et par l'emploi généralisé du carrelage au sol et aux murs, une caractéristique des zoos de cette époque, qui leur donne un air d'hôpital du fait d'un hygiénisme débridé et de la volonté de faciliter un peu plus le travail d'entretien.

L'artifice fut encore plus sensible à propos de la semi-liberté, qui en réalité ne concernait pas les animaux toujours parqués mais les spectateurs. Il s'agissait de leur donner «l'impression que les bêtes sont en liberté », de créer une apparence d'affranchissement ou tout au moins une sensation d'emprisonnement moindre. Deux faits montrent l'importance de cet aspect visuel dans les nouvelles installations. En 1967, au moment des discussions sur le projet de giraferie, le directeur du zoo s'opposa à l'architecte, non pas à propos des conditions d'hébergement des animaux, mais sur le fait que le bâtiment prévu ne s'intégrait pas au paysage, qu'il ne permettait pas une bonne observation des cages internes, et sur la tentation, ensuite abandonnée, d'installer des grillages moins onéreux que les fossés. À la fauverie, un local

la fauverie, 1973.

86. Qui a publié de nombreux livres sur le sujet, depuis: Heini HEDIGER, Les

, animaux sauvages en captivité, introduction à la biologie desjardins zoologique (1943), Paris, Payot, 1953, jusqu'à Heini HEDIGER, Lieu de naissance,' zoo, Zurich, Silva, 1984. 
aseptisé, on disposa des lampes à sodium pour préserver la perception des couleurs des pelages! 87

Il s'agit bien d'une «politique de présentation », comme l'écrit l'architecte de la fauverie en 1973, d'un procédé théâtral, comme le suggère l'expression - inconsciente? - de «plates-formes» utilisée pour désigner les nouveaux enclos. À l'époque d'Hagenbeck et de Vincennes, ce dispositif avait eu pour but de créer un exotisme plus vrai. Si l'idée restait présente dans le cas lyonnais, elle était désormais marginale à l'heure du tourisme de masse et de la télévision, et cédait la place à deux autres objectifs. Il fallait d'abord donner l'impression aux spectateurs «que rien ne les sépare» des animaux 88. On voulait ainsi être en phase 89 avec le nouveau regard sur le monde sauvage, qui s'était composé peu à peu au $\mathrm{XX}^{\mathrm{e}}$ siècle, d'une manière irrégulière selon les pays et les régions, sans qu'on puisse en faire ici la genèse, et qui s'imposa à partir des années 1960 sous l'impulsion - ou comme en témoignent - de nombreux livres, de films à la mode Cousteau, d'émissions de télévision. Il se caractérise par une valorisation du sauvage, un reflux de la peur et de l'idée de férocité, une volonté de connaitre, d'être proche et non opposé. Il s'inscrit, avec le processus concomitant de décolonisation, dans un phénomène de remise en cause du rôle de l'Occident dans le monde, qui devrait désormais comprendre, respecter et non plus asservir.

L'autre objectif était de donner une forte image de nature et de liberté pour contrer les critiques croissantes sur le zoo prison 90. Autrement dit, il s'agit d'une adaptation à l'évolution des mentalités, à une autre vision de la nature. Mais en même temps, ce type de zoo, qui s'est répandu en Occident, symbolise, avec sa nature composée, artificielle, avec son cheptel à la démographie bien contrôlée, toute l'ambition de l'homme du $\mathrm{XX}^{\mathrm{e}}$ siècle, ce nouveau démiurge, face à la nature: la conserver parce qu'il ressent un besoin du sauvage, mais aussi la prendre en main, la gérer et la façonner à sa guise.

87. Arch. mun. Lyon, 849 WP liasse 434, Directeur au maire, 17 octobre 1967; 852 WP liasse 575/2, Rapport de l'architecte sur la fauverie, 1973. La citation est tirée de ce dernier texte.

88. Sur les objectifs: Arch. mun. Lyon, , 852 WP liasse 575/2, Rapport de l'architecte, 1973 ; "Le parc de la Tête d'Or", dans Lyon.Horticole, ouv. cité, pp. 36-39 (seconde citation).

89. On peut dire aussi que l'aménagement résultait de ce nouveau regard et le reflétait, les intervenants, des directeurs aux architectes, le partageant à des degrés divers.

90. Mais ce fut un échec, car ces réalisations, déjà à la traîne chronologiquement par

rapport aux autres zoos européens, apparurent vite dépassées: les années 1960-1970 virent l'ouverture en France des grands parcs-safaris (Thoiry, Peaugres ...) qui apparurent à beaucoup comme la nouvelle référence. 